\title{
Three cell flying capacitor inverter for dielectric barrier discharge plasma applications
}

\author{
A. Flores-Fuentes ${ }^{a}$, R. Peña-Eguiluz ${ }^{b},{ }^{*}$ López-Callejas $^{a, b}$, A. Mercado-Cabrera ${ }^{b}$, \\ R. Valencia-Alvarado ${ }^{b}$, S. R. Barocio ${ }^{b}$, and A. de la Piedad-Beneitez ${ }^{a}$ \\ a Instituto Tecnológico de Toluca, Av. Tecnológico S/N, \\ A.P. 890, Toluca, México and \\ ${ }^{b}$ Instituto Nacional de Investigaciones Nucleares, Plasma Physics Laboratory, A.P. 18-1027, C.P. 11801, México D.F., México
}

(Received on 25 November, 2008)

\begin{abstract}
It is reported the design, construction and initial tests of a three cell flying capacitor inverter (TCFCI) in a half-bridge configuration. The device operates at a $200 \mathrm{kHz}$ frequency which leads to a voltage output at 12.5 $\mathrm{kHz}$ presenting an acceptable response in an open-loop configuration. These features outdo those reported in the current multilevel converter literature. The TCFCI is driven by pulse width modulation, following a phase shift (PS-PWM) control strategy, in order to generate a steady AC voltage signal. This inverter is used to excite a dielectric barrier discharge cell (DBDC) intended for cold plasma generation at room pressure. Some results obtained for two different kinds of atmosphere, helium and argon, are presented. All the system having been tested, early recorded voltage and current waveforms, are included. Finally, three methods for calculating the related electric efficiency of the discharge cell are discussed.
\end{abstract}

Keywords: DC-AC power converters, multilevel inverters, electric discharges, plasma

\section{INTRODUCTION}

Dielectric barrier discharges (DBD) also known as silent discharges, are commonly produced in parallel plates or coaxial cells at near atmospheric pressure (1-3 atm). Plasmas in a dielectric barrier discharge cell (DBDC) are generated by applying high voltage (HV) to a pair of electrodes, separated by a gap, where at least one of these is covered by a dielectric sheet, usually made of quartz, glass or ceramic. These discharges are frequently used in industrial ozone generation [1] or in the treatment of air pollutants [2], in particular, solvents or volatile organic compounds (VOC) which are mainly produced around chemical plants [3]. The conditions required to produce non-equilibrium plasmas are easier to attain with DBDC than by alternative methods such as low pressure discharges, high pressure discharges with fast pulses, or electron beam injection. DBD are generated in a high pressure gas and consist of a multitude of short lived current filaments [4]. Due to the short duration of these micro discharge channels and the prevailing low ion mobility, the kinetic energy in silent discharges is principally transferred to electrons, which become hot, unlike the other species. This behavior provides a significant electronic excitation of molecules. As a consequence, the plasma promotes efficient thermo chemical reaction processes, which are desirable in the destruction of hazardous organic compounds [5]. In order to achieve a stable DBD performance, the incumbent power sources usually provide $\mathrm{HV} \mathrm{AC}$, from some tens of $\mathrm{Hz}$ [6] up to the order of several $\mathrm{kHz}$ [7] with amplitudes from 1 to $100 \mathrm{kV}$. Thus, several authors have resorted to resonant inverters either in a halfbridge configuration [8] or in a full-bridge one, operating at tens of $\mathrm{kHz}$ [9]. Nevertheless, these converters, however efficient, are constrained to operate in a narrow frequency range.

The present report presents some early results of a complex system which is mainly constituted by a DBDC made of parallel plates biased by a coupled transformer whose primary is driven by a voltage source inverter (VSI) configured on

*Electronic address: rosendo. eguiluz@inin.gob.mx the basis of a half-bridge three cell flying capacitor inverter (TCFCI). Pulse width modulation by means of phase shifting (PS-PWM) has been applied in order to drive the TCFCI. The latter has been implemented through a hybrid electronic circuit, which generates six commutation pulses for the high power switches that constitute the TCFCI. An appropriate operating system provides a four-level chopped voltage at the output inverter. The correct performance of the control strategy enables a good open-loop inverter behavior due to a natural balance originated in the inherent current harmonics. An electrical characterization of the system, including efficiency calculations, has been included.

\section{SYSTEM DESCRIPTION}

The electric circuit representation of the proposed DBD system is shown in Fig. 1. It is mainly composed by a variable autotransformer supplying an adjustable $\mathrm{AC}$ voltage level within $0-220 \mathrm{~V}$ to a non controlled full wave rectifying bridge. It delivers in turn a DC voltage to a voltage divider constituted by two capacitors, $C_{S 1}$ and $C_{S 2}$, along with a $C_{S f}$ one, functioning like input filter. Thus, a bipolar DC voltage is applied to the input of a TCFCI which is assembled by means of three commutation cells. Each one of the last is implemented with a couple of complementary switches, namely: $\left(T_{1}, T_{1}{ }^{\prime}\right),\left(T_{2}, T_{2}{ }^{\prime}\right)$ and $\left(T_{3}, T_{3}^{\prime}\right)$ respectively. These are highfrequency high-voltage MOSFET's associated to free wheeling diodes and separated by a floating voltage source. Each one of the flying capacitors $C_{1}$ and $C_{2}$ are connected between two different commutation cells. The capacitors operate as an ideal voltage supply whereby the averaged current must vanish. It is accomplished when the inverter maintains a balanced voltage distribution between these capacitors by applying identical work cycles to the switches. The number of capacitors determines directly the amount of voltage levels that the converter is able to handle. Assuming an identical capacitance: $C=C_{1}=C_{2}$, then the voltage across each capacitor $V_{C_{k}}$ is given by $V_{C_{k}}=\frac{k}{p} V$ [10], where: $V$ is the DC input voltage, $p$ is the number of cells and, $k=1, . ., p-1$, is the cell position. In this paper, the voltage of each flying capacitor is 
defined by $V_{C_{1}}=\frac{1}{3} V$ and $V_{C_{2}}=\frac{2}{3} V$ respectively. Meanwhile, the capacitance value can be determined as:

$$
C_{k}=\frac{I_{S \max }}{p f_{S W} \Delta V_{C_{k}}}
$$

where: $\Delta V_{C_{k}}$ is the maximum permissible ripple voltage provided by the floating capacitor, $I_{S \max }$ is the maximum DC current inverter and, $f_{S W}$ is the switching frequency. A converter operation guaranteeing open loop stability is determined by two main factors: (i) an adequate sequence of the commutation pulses imposed to the power switches and (ii) the correct effective voltage levels $V_{C_{k}}$ among flying capacitors.

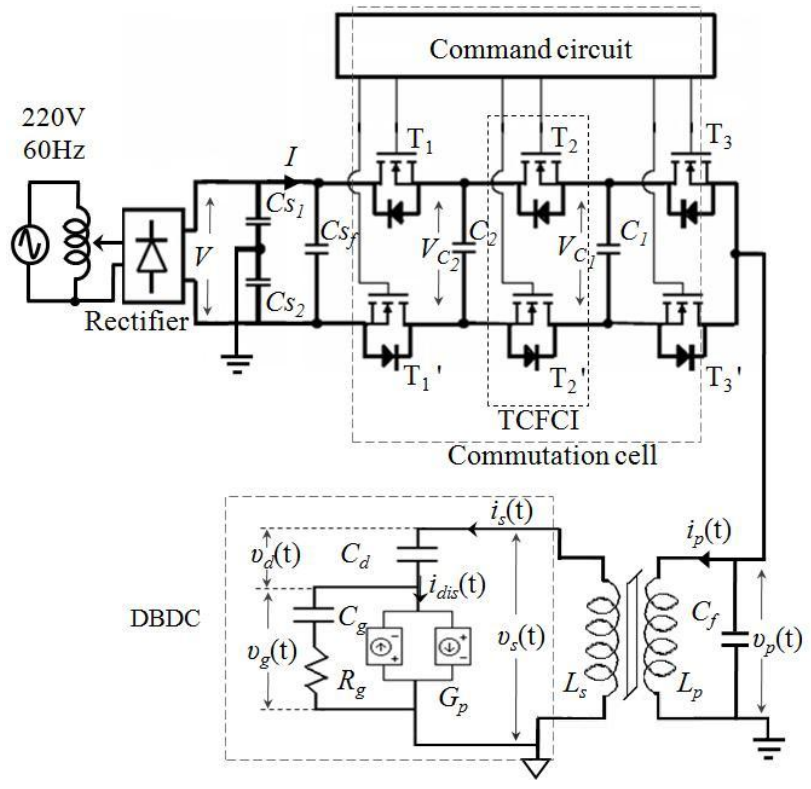

FIG. 1: Diagramme of the implemented DBD system

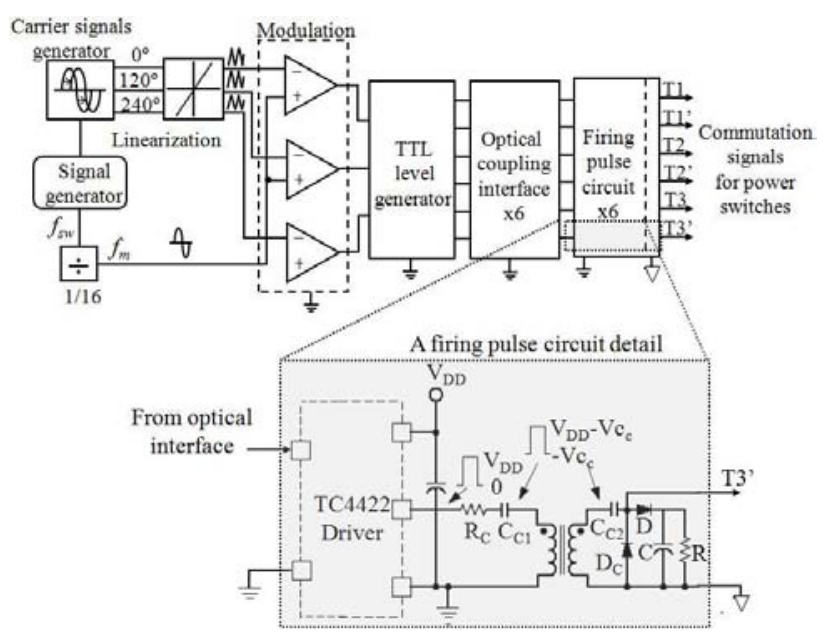

FIG. 2: Block diagram of the implemented PS-PWM technique and a detailed conditioning switch gate pulse circuit

The TCFCI output voltage is applied to the DBDC, herein modeled by a parallel circuit composed by a capacitor $C_{f}$ and the primary winding of an isolation step-up (1:30) HV transformer. The proposed electric model of the DBDC is characterized by: $C_{d}$ which represents the equivalent capacitance of the dielectric sheets placed in both electrodes. $C_{g}$ is the gas capacitance, $R_{g}$ is the plasma sheet resistance, and $G_{p}$ is a voltage-controlled current source reproducing the exponential growth of the discharge current $i_{d i s}(t)$ when the voltage across the cell, $v_{s}(t)$, surpasses the breakdown threshold voltage $V_{b} . C_{s}$ is the stray capacitance present in the real equipment.

A PS-PWM strategy was chosen in order to modulate the pulses width applied to VSI power switches as seen in Fig. 2. The system is supported by a sinusoidal waveform generator which provides a reference $f_{S W}=200 \mathrm{kHz}$ signal to an operational amplifiers stage, where three $\frac{2}{3} \pi$ out of phase sinusoidal signals are generated. The latter are applied to a logarithmic amplifier stage, which in turn produces triangular waveforms meant to act as carrier signals to be compared to the modulating one (produced by a digital frequency divider, and later on integrated, so to obtain a sinusoidal waveform with frequency $\left.f_{m}=\frac{1}{16} f_{S W}=12.5 \mathrm{kHz}\right)$. The modulation process is developed by comparing the modulating sinusoidal signal and the triangular waveforms, resulting in three different sequences of pulses which are conditioned in order to meet TTL levels. After that, the resulting pulses are optically isolated between the command circuit and the power switches by means of optic fiber link connections reducing the electromagnetic interference (EMI). Likewise, another circuit has been implemented with a magnetic coupling provided that the firing signal of each power switch should be applied with respect to a floating voltage. Thus, driver outputs are coupled by means of pulse transformers and commutation signals, given that the switches need additional components, as it is shown in the firing circuit detail of Fig. 2. There, capacitor $C_{C 1}$ provides the restoration voltage for the inductance of the primary winding transformer; both of these components integrating an $L C$ circuit. Thus a resistor $R_{C}$ is added with the purpose of preventing any resonant effect. Additionally, capacitor $C_{C 2}$ along with diode $D_{C}$ are used to re-establish the driver's original amplitude. Finally, the circuit containing capacitor $C$, resistor $R$ and diode $D$ modifies the dynamical behavior of the pulse signal provided from the secondary winding of the pulse transformer.

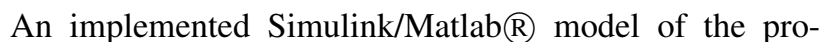
posed system makes possible to obtain a valuable approaching of its dynamic performance. As the simulation outcome in Fig. 3.a depicts, three triangular mutually $\frac{2}{3} \pi$ out of phase signals are compared independently from the modulating sinusoidal signal at the frequency $f_{m}$. When the modulating amplitude exceeds that of the carrying signal, a voltage pulse is generated in order to start the power switch and therefore increase the power supply. The resulting pulse sequence is presented in Fig. 3.b. On its part, Fig. 4.a displays the voltage output of the TCFCI in vacuum in order to demonstrate that it is actually modulated by three different voltage levels imposed by the flying capacitors. In addition, the modulated voltage applied to the primary winding generates a quasisinusoidal current waveform. Thus, the electric energy transferred to the secondary winding increases the input voltage so as to get a well established discharge. As it can be observed in Fig. 4.b, the discharge current and voltage waveform resulting from the simulation closely resemble the archetypal discharges obtained experimentally as in [11]. 

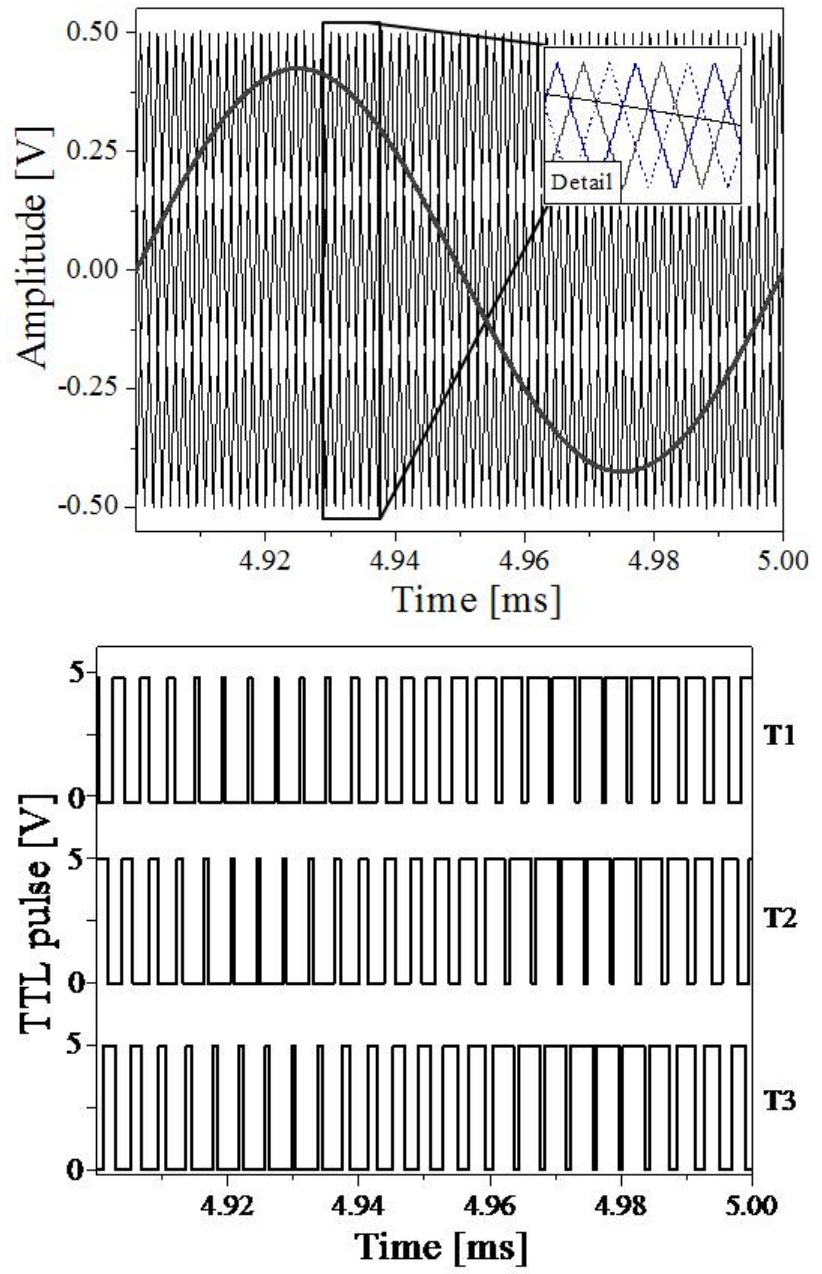

FIG. 3: Simulation outcome from the implemented modulation strategy circuit: (a) the three carrier signals are compared with respect to the modulating signal; (b) resulting sequences to be applied as control signals to the power switches

\section{EXPERIMENTAL SET-UP}

The instrumental arrangement used in this study is represented in Fig. 5, where several photos of previously described TCFCI circuits can be identified. A top down view of the implemented DBDC is depicted in Fig. 6.a; meanwhile, Fig. 6.b illustrates the inner cell structure. The DBDC is constituted by two electrodes of $0.12 \mathrm{~m}$ diameter made of aluminum separated by two glass dielectric sheets, $0.002 \mathrm{~m}$ thick, and a gap $l=0.002 \mathrm{~m}$ between them. Thus, the total cell capacitance of the DBDC is given by an equivalent capacitance $C_{d}=\varepsilon_{0} \varepsilon_{r} S / 2 l$, where the industrial glass dielectric relative permittivity is $\varepsilon_{r}=4.9, \varepsilon_{0}=8.8541 \times 10^{-12} \mathrm{~F} / \mathrm{m}$ is the permittivity in vacuum, and $S=0.0113 \mathrm{~m}^{2}$ is the total calculated electrode surface. Hence, the DBDC calculated capacitance turns out to be $C_{d}=122 \mathrm{pF}$. The gas capacitance $C_{g}$ was calculated for argon and helium, in both cases its magnitude being near $50 \mathrm{pF}$. The DBDC gas supply was endowed with a pipeline connected from two gas containers through a gas mixer and mass flow meters. In all cases, electric discharges were generated at atmospheric pressure with gas mass flow of 2.0 LPM.
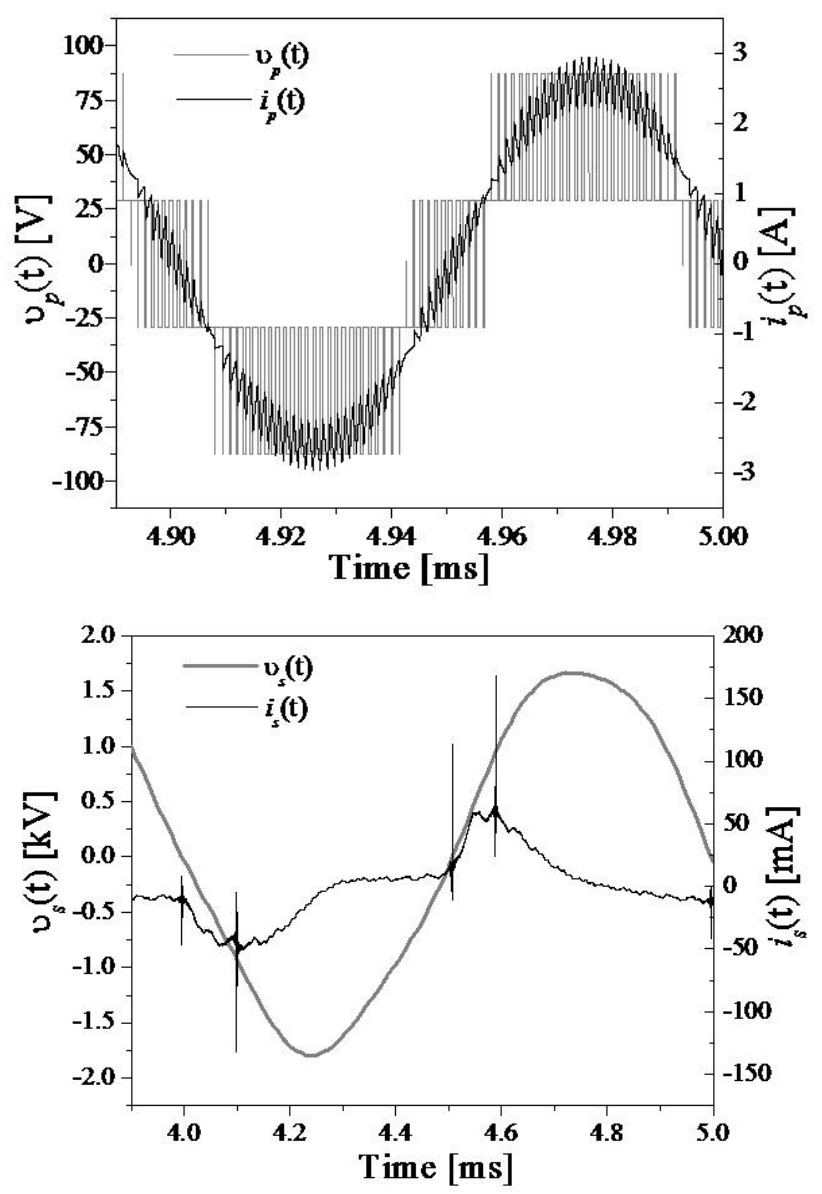

FIG. 4: Simulation outcome of the primary winding voltage supply and resulting current intensity. (b) Applied cell voltage and its resulting current in the case of an argon discharge

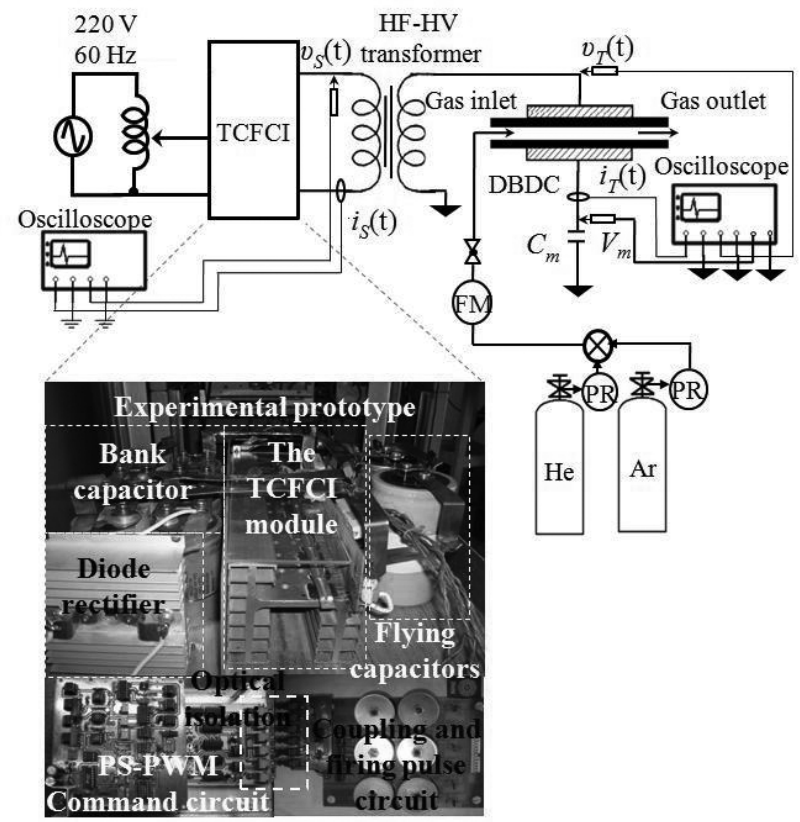

FIG. 5: Block scheme of the experimental layout 


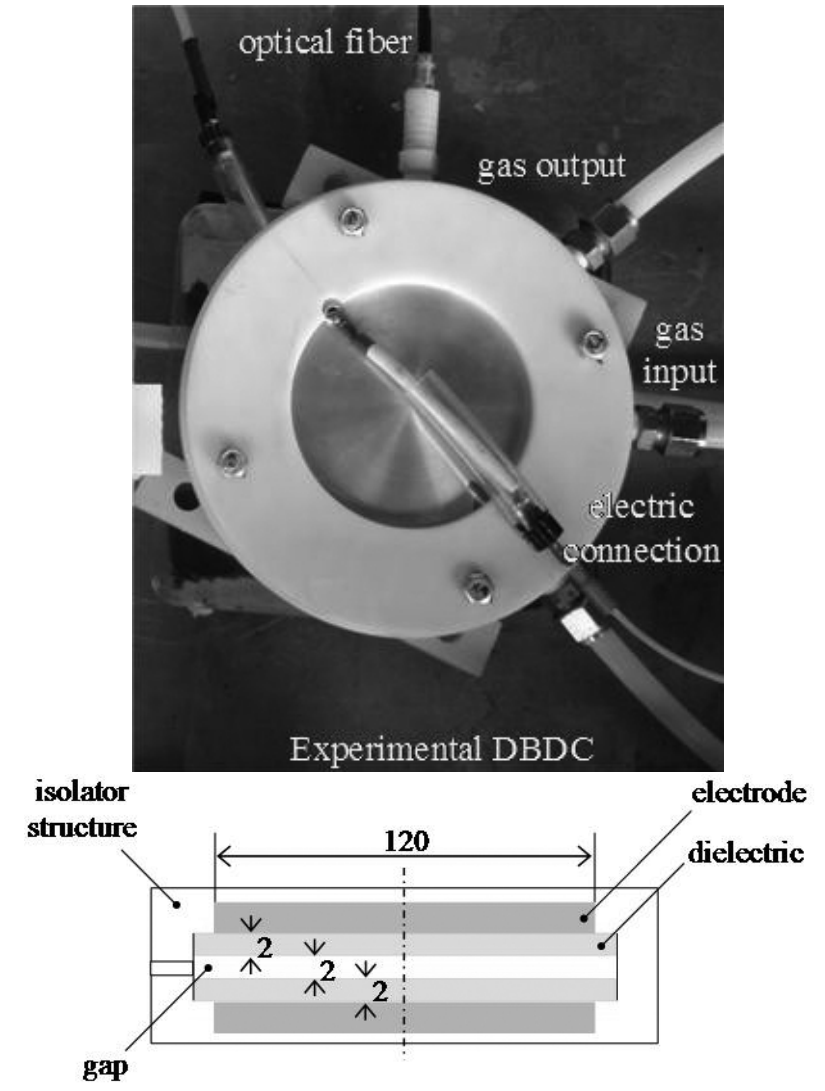

Units are in mm

FIG. 6: (a) Top down view of the operational DBDC, and (b) schematic representation of the DBDC cross section

The TCFCI output voltage and current $\left(v_{p}(t), i_{p}(t)\right)$ and DBDC applied voltage and discharge current $\left(v_{s}(t), i_{s}(t)\right)$ are monitored by means of a (Tektronix TDS2014) oscilloscope equipped with a HV probe (Tektronix P6015) and a current sensor (Stangenes model 0.5-1.0 W). We consider as an illustration the DBDC applied voltage and its resulting current waveform $\left(v_{p}(t), i_{p}(t)\right)$ captured during the stable phase of the discharge, shown in Fig. 7. From these waveforms, the peak values of $v_{p}$ about $28 \mathrm{~V}$ and of $i_{p}$ about $5.8 \mathrm{~A}$ have been determined. Thus, real power applied to the primary winding of the transformer is given by:

$$
P_{p}(t)=v_{p r m s}(t) \cdot i_{p r m s}(t) \cdot \cos (\theta)
$$

which, considering that measured value of $\theta=85^{\circ}$, amounts to $P_{p}(t) \approx 7.1 \mathrm{~W}$ : an expected result as the DBDC exhibits a predominantly capacitive conduct.

Fig. 8 portrays the voltage and current wave forms measured at the DBDC plugs. The $v_{s}(t)$ peak value reaches about $1.75 \mathrm{kV}$ while that of $i_{s}(t)$ is $5 \mathrm{~mA}$, estimated from its first harmonic. Although the instantaneous power absorbed by the DBDC can be directly assessed from the product of $v_{s}(t)$ and $i_{s}(t)$, it can also be assessed from the charge stored in the cell, $Q_{s}(t)$ (here represented by the measured voltage $v_{m}(t)$ of a reference capacitance $C_{m}$, keeping in mind that $\left.Q_{s}(t)=C_{m} v_{m}(t)\right)$ versus the applied voltage, $v_{s}(t)$ (Fig. 9) [12],[13]. In this work we considered that $C_{m} \sim 2 \mu \mathrm{F}$ is the reference capacitance shown in Fig. $5, V_{m}$ is the voltage am-

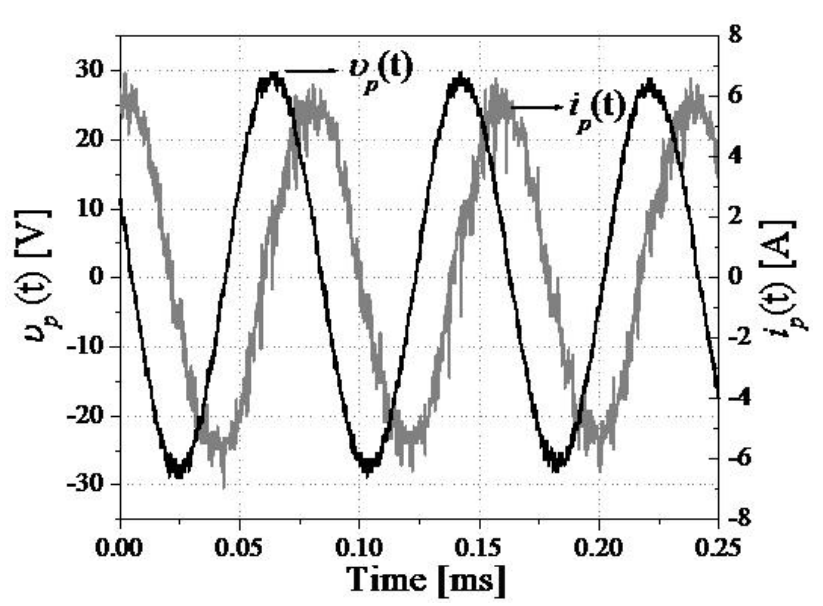

FIG. 7: Experimental waveforms of the applied voltage $v_{p}(t)$ and of the resulting current intensity $i_{p}(t)$, in the case of a 2 LPM argon flow at room pressure.

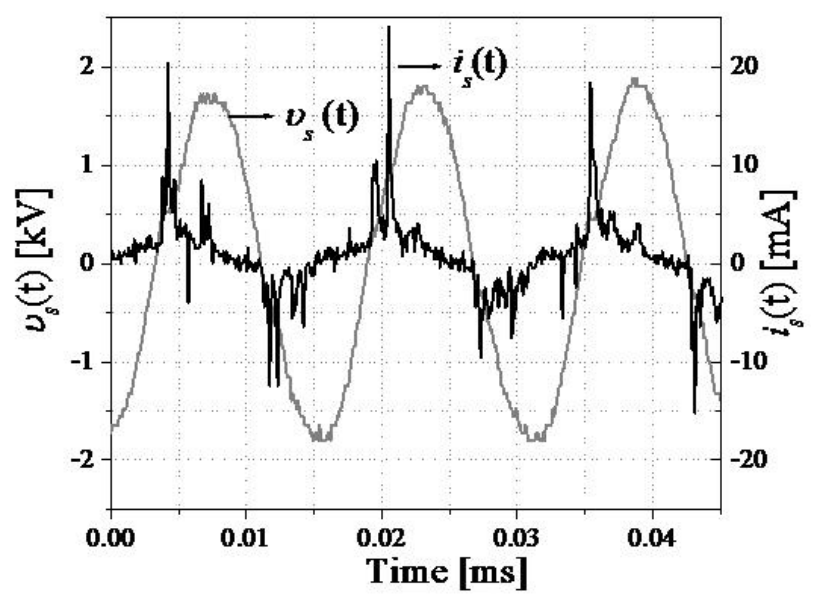

FIG. 8: Waveforms of $v_{s}(t)$ and $i_{s}(t)$ at the DBDC operating with a 2 LPM argon flow at room pressure.

plitude per division on the vertical axis of Fig. 9, namely 20 $\mathrm{mV} / \mathrm{div}$, and $V_{s} 1 \mathrm{kV} / \mathrm{div}$ is the magnitude of a division on the horizontal axis. Thus, the computed energy stored by the cell $E=0.196 \mathrm{~mJ}$ while the power supplied to the cell $P_{C}=f E$, where $f$ is the frequency of the excitation signal applied to the DBDC, namely, $P_{C}=2.45 \mathrm{~W}$. In other words, the power transferred from the transformer secondary to the cell attains an electric efficiency of $34.5 \%$ in the case of argon discharges.

The $v_{p}(t)$ peak level, which is about $12.5 \mathrm{~V}$, and the corresponding $i_{p}(t)$ peak, around $3 \mathrm{~A}$, have been obtained from the characteristic signals applied to the transformer primary as seen in Fig. 10 in the case of a helium discharge. Thus, the real power applied to the primary winding, considering that $\theta=85^{\circ}$ leads to a value $P_{p}(t) \approx 2.61 \mathrm{~W}$. The signal in the DBDC is depicted in Fig. 11. The peak value of $v_{s}(t)$ is around $1 \mathrm{kV}$, and, approaching $i_{s}(t)$ by its first harmonic, its maximal value reaches $2 \mathrm{~mA}$.

Therefore, as $v_{m}(t)=Q_{s}(t) / C_{m}$ then it is possible to get the charge stocked $Q_{s}(t)$ in the reactor. Thus, in the case of helium discharges from the $v_{m}(t)$ versus $v_{s}(t)$ characteristic as 


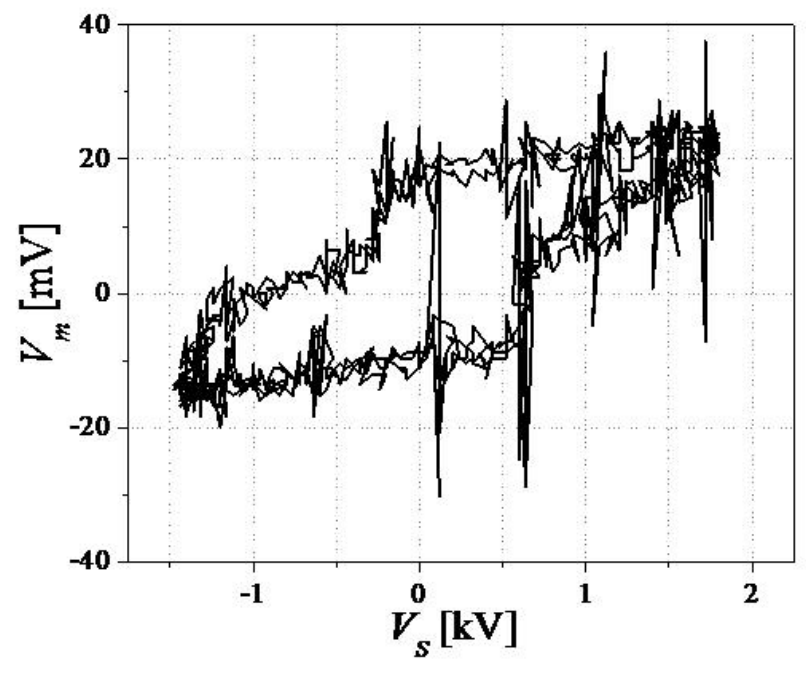

FIG. 9: $v_{m}(t)$ versus $v_{s}(t)$ Lissajous wave form in the DBDC with a $12.5 \mathrm{kHz}$ excitation frequency when fed with argon.

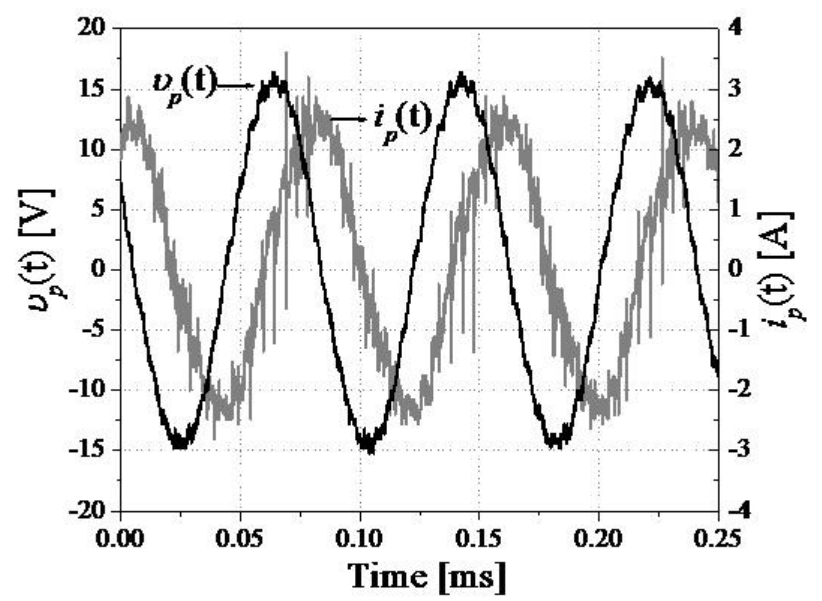

FIG. 10: Experimental time patterns of the applied voltage $v_{p}(t)$ and its associated current $i_{p}(t)$, in a 2 LPM flow of helium at room pressure

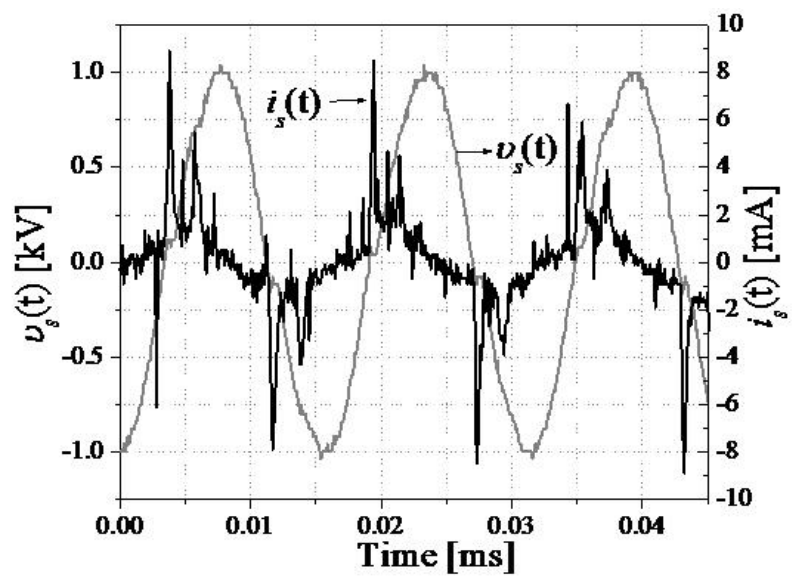

FIG. 11: $v_{s}(t)$ and $i_{s}(t)$ time patterns measured in the DBDC, working on a 2 LPM helium flow at room pressure the one shown in Fig. 12, then $V_{m}=10 \mathrm{MV} / \mathrm{div}, V_{s}=1 \mathrm{kV} / \mathrm{div}$, whereby $E_{C}=20 \mu \mathrm{J} / \mathrm{div}^{2}$ and, consequentially, $E=0.045 \mathrm{~mJ}$. Finally, $P_{C}=0.562 \mathrm{~W}$ so that the power transferred from the primary to the cell can be estimated as $21.53 \%$.

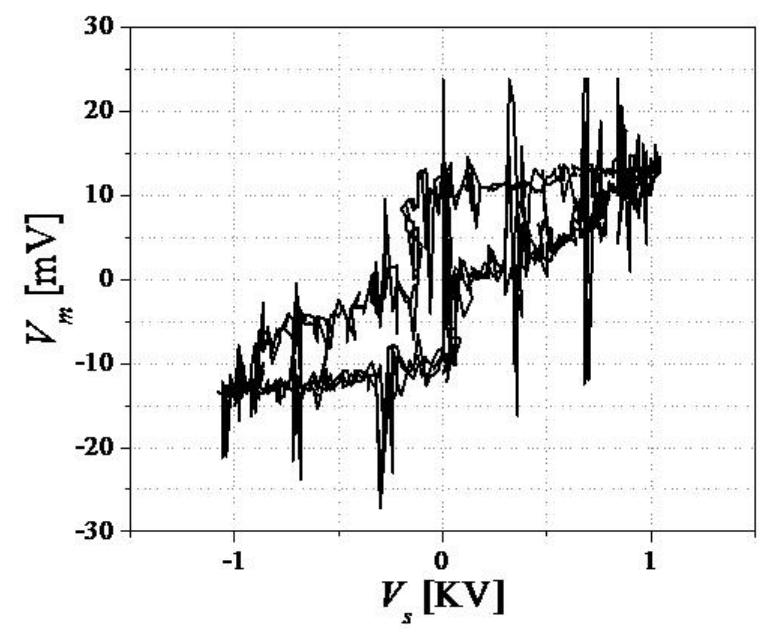

FIG. 12: $v_{m}(t)$ versus $v_{s}(t)$ waveform of a DBDC with helium and a $12.5 \mathrm{kHz}$ excitation frequency

A second method for calculating the power consumed by the DBDC yields the following Manley expression [13]:

$$
P_{c}=4 V_{b} C_{d} f\left[V_{M}-V_{b}\left(\frac{C_{d}+C_{g}}{C_{d}}\right)\right]
$$

where: $V_{M}$ is the maximal amount of voltage supplied to the cell. Consequentially, operating it on argon requires $2.64 \mathrm{~W}$ which implies an $34.6 \%$ efficiency while, in helium, the required power, $0.564 \mathrm{~W}$, improves the efficiency up to $21.6 \%$.

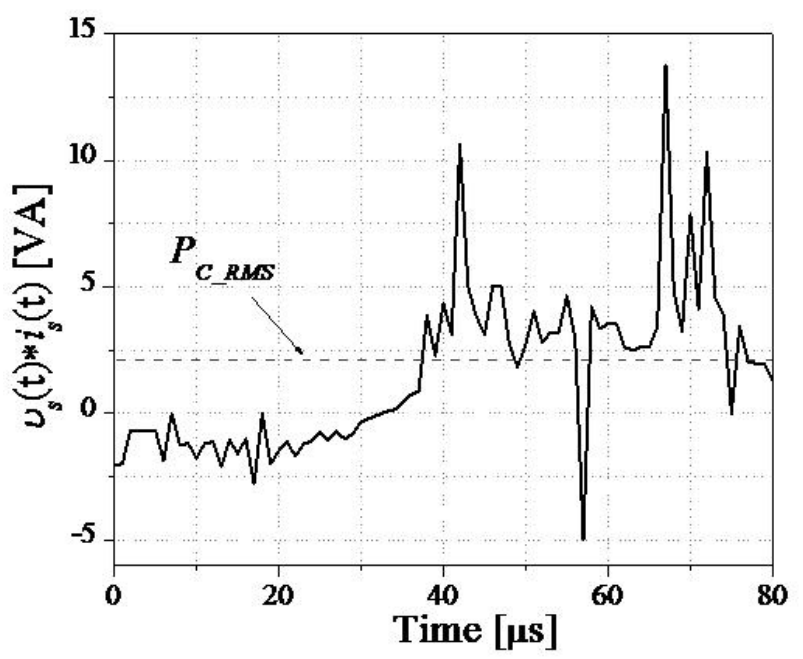

FIG. 13: Waveforms of the instantaneous power $v_{s}(t) \cdot i_{S}(t)$ applied to the DBDC with a $21 / \mathrm{min}$ argon flow at room pressure

A final analysis of the instantaneous power of the signals applied to the DBDC, that is, voltage and current intensity at the secondary of the step up transformer, enables one to obtain the actual value of the applied power in both gases, as 


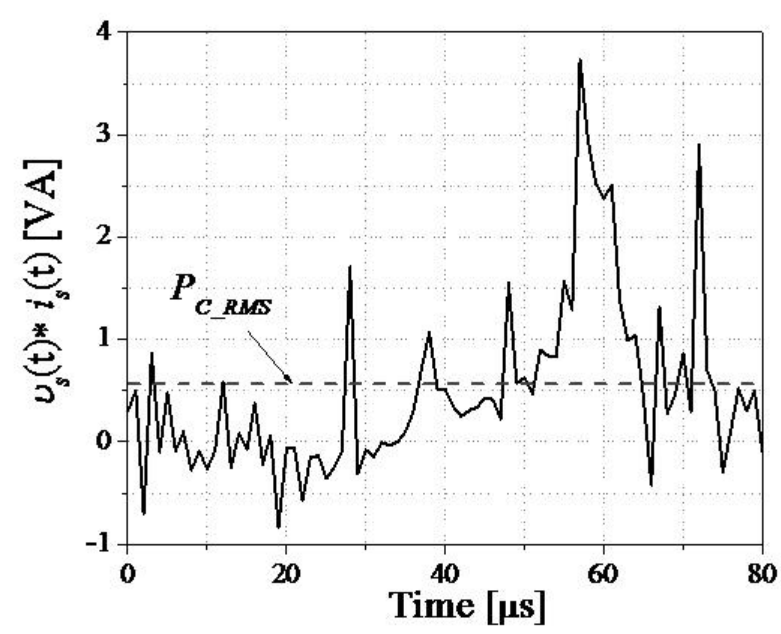

FIG. 14: Waveforms of the instantaneous power $v_{s}(t) \cdot i_{S}(t)$ applied to the DBDC with a $21 / \mathrm{min}$ helium flow at room pressure.

suggested by Fig. 13 (argon) and Fig. 14 (helium). In a similar fashion, Table I gathers the results of the power estimation by all three methods including the respective efficiency values with both gases.

It is obvious that the first and third methods seem to agree better, which could be expected from their being obtained from experimental values. In contrast, the second one depends on the breakdown voltage, rather roughly estimated from the experimental conditions.

\section{CONCLUSIONS}

The design TCFCI built out of high power high frequency semiconductors intended to generate room pressure dielectric barrier discharges has been described. Its hybrid system of command consists of a relatively simple and stable cycle which simplifies the handling of reference signals in order to accomplish the required power switch commutation through intermediate circuits able to isolate the low power stage from the high one. Thus, the use of an insulation stage reduces electromagnetic interference significantly along with the transients typical of the high power systems.

The open loop operation of the proposed system maintains a stable behaviour conducive to a well ordered voltage distribution in the floated capacitors given the selected modulation technique. Although the proposed design allows one to adjust the frequency of the reference sinusoidal signal so to modify the DBDC excitation frequency, the $12.5 \mathrm{kHz}$ frequency was maintained throughout the study provided that this is the maximal possible value for the whole system. Thus, when a different modulation system is in place, e.g., a square pulse one, it is possible to raise the frequency even further with the consequent improvement in the general efficiency, albeit with some change of components.

The static converter developed for the DBDC discharge generation has been satisfactorily tested by establishing room pressure discharges in a parallel plate cell containing helium and argon, achieving efficiencies above 20 and 30 respectively. An innovative conversion characteristic of the proposed TCFCI is its high $12.5 \mathrm{kHz}$ operation frequency, far beyond the $3 \mathrm{kHz}$ one reported in similar inverters.

\begin{tabular}{|c|c|c|c|c|c|c|}
\hline Gas & \multicolumn{2}{|c|}{ Rhomboid method } & \multicolumn{2}{c|}{ Manley's equation } & \multicolumn{2}{c|}{ Real power } \\
\hline argon & $2.45 \mathrm{~W}$ & $32.11 \%$ & $2.64 \mathrm{~W}$ & $34.6 \%$ & $2.34 \mathrm{~W}$ & $30.66 \%$ \\
\hline helium & $0.562 \mathrm{~W}$ & $21.53 \%$ & $0.564 \mathrm{~W}$ & $21.6 \%$ & $0.575 \mathrm{~W}$ & $22.03 \%$ \\
\hline
\end{tabular}

TABLE I: Comparison of power values applied to the DBDC and their resulting efficiency according to three distinct calculation procedures

\section{Acknowledgements}

This work received partial financial support from CONACYT and DGEST, Mexico. The authors are very grateful for the technical collaboration received from M. T. Torres M., I. Contreras V. and P. Angeles E.
[1] C. Gottschalk, J. A. Libra and A. Saupe, Ozonation of Water and Waste Water: A Practical Guide to Understanding Ozone and its Applications, Wiley-VCH; Weinheim (2000).

[2] C. H. Wang, G. F. Li, Y. Wu, Y. Wang, J. Li, D. Li and N. H. Wang, Plasma Chem. Plasma P. 27, 1, 65 (2007).

[3] O. Godoy-Cabrera, R. López-Callejas, R. Valencia, A. MuñozCastro, S. R. Barocio, E. Chávez A., A. Mercado-Cabrera, A. de la Piedad-Beneitez, B. Rodríguez-Méndez and J. Rodríguez-Arce, Braz. J. Phys. 34, 4B, 1766 (2004).

[4] U. Kogelschatz, B. Eliasson and W. Egli, J. Phys. IV France, 1997, 7, C4, 47 (1997).

[5] L. A. Rosocha, Processing of Hazardous Chemical Using Silent-Discharge Plasmas, in Environmental Aspects in Plasma Science, Ed. W. Manheimer,American Institute of Physics Press, New York, (1997).

[6] J. Velikonja, J. A. Robinson, M. A. Bergougnou, J. S. PeterCastle, W. L. Cairns and I. I. Inculet, Proc. IEEE International Symposium on Electrical Insulation, Anaheim CA, USA, 2-5
April, 1, 25 (2000).

[7] D. Evans, L. A. Rosocha, G. K. Anderson, J. J. Coogan and M. J. Kushner, J. Appl. Phys. 74, 9, 5378 (1993).

[8] O. Godoy-Cabrera, J. S. Benítez-Read, R. López-Callejas and J. O. Pacheco-Sotelo, Int. J. Electron. 87, 3, 361 (2000).

[9] O. Godoy-Cabrera, J. S. Benítez-Read, R. López-Callejas, J. O. Pacheco-Sotelo and A. de la Piedad-Beneitez, Int. J. Electron. 92, 6, 327 (2005).

[10] F. Hamma, T. A. Meynard, F. Tourkhani and P. Viarouge, Proc. IEEE Power Electronics Specialists Conf. (PESC '95), Atlanta, USA, 18-22 June, 2, 1208 (1995)

[11] N. Naude, J. P. Cambronne, N. Gherardi and F. Massines, J. Phys. D: Appl. Phys. 38, 530 (2005).

[12] Z. Falkenstein and J. J. Coogan, J. Phys. D: Appl. Phys. 30, 817 (1997).

[13] T. C. Manley Trans. Electrochem. Soc. 84, 83 (1943). 